\title{
How to create a summary of findings table with GRADEPro
}

\author{
Arindam Basu ${ }^{1}$ \\ 1 University of Canterbury \\ Funding: The author(s) received no specific funding for this work.
}

Potential competing interests: The author(s) declared that no potential competing interests exist.

\begin{abstract}
GRADE provides a structure for clinicians and healthcare analysts to understand the quality of evidence for an outcome. An outcome can be associated with a number of studies, and quality appraisal in terms of whether additional evidence must be needed before a definitive conclusion about an intervention-outcome can be arrived at is done on the basis of conducting GRADE tables. GRADEpro as a software enables construction of grade tables, and has two parts. In part one of this series, we showed how to construct certainty of evidence tables. In this part, we discuss how to create summary of findings tables with GRADEpro for dichotomous outcome variables.
\end{abstract}

\section{Introduction}

In the previous article in this series, we presented a step by step process of entering data to GRADEpro to appraise a body of evidence. There, we have shown that you can enter data to GRADEpro and conduct an appraisal of the certainty. In evidence based health and in GRADE process (GRADE is a shorthand form of Grading Recommendations for Appraisals and Development of Evaluations), the focus is on using outcomes as a first principles of critically appraising evidence [1]. In appraising the quality of evidence, a study can include a number of outcomes; likewise an outcome can be studied by a number of studies. Hence, the focus is on specific outcomes that we would like to study.

In that process, here, we are going to focus on how to use GRADEpro to correctly write a summary of findings table. The summary of findings table is the second part of the evidence portfolio; the first part of evidence portfolio is the uncertainty assessment where for each outcome and outcome measurement, we assessed what was the study design, what biases were identified, whether the studies were consistent in what they found, and other issues. In the end, we came to a conclusion whether we would need more information about the association between the intervention and outcomes so that more studies would be necessary or whether the current body of studies would be sufficient in themselves to arrive at a conclusion whether an intervention I works to achieve an outcome $\mathrm{O}$.

The summary of findings here help to quantify the association we observe. As before, let's start with an article and work step by step.

Here we have selected an article by Philip Tonneson et.al. (2012) where they evaulated the efficacy of nicotine mouth spray for smoking cessation in a randomised double blind trial ${ }^{[2]}$. Obtain the full text of this article and follow along. 


\section{What is the outcome they measured in this study?}

They measured the outcome as "Continuous abstinence"

\section{How was that outcome measured?}

From the article, we get to see that

Continuous abstinence was defined as self-reported "no smoking", verified by a CO value ,10 ppm, from week 2 up to and including the given visit. Any subject who missed the visit(s) at week(s) 8, 16 and/or 20, or for some other reason had missing CO value(s) at one or more of these visits, was not regarded a treatment failure if the subject was verified continuously abstinent at a later visit.

(See pp 550, in the Statistics section)

This should tell you that "continuous absence" is a binary variable.

And why so?

Think of yourself as the study investigator. If you asked a participant whether she maintained "no-smoking" status and the participant replied, "yes", and then you verified that the participant was indeed "non-smoking" status using the CO value, then you would either

(1) label that person as a non-smoker or

(2) if the participant lied and failed the CO test, you'd label that person as "smoker".

There are TWO choices, hence this is a dichotomous variable ("binary" means two).

Then ....

The intervention was "Nicotine mouth spray".

How many people were in the intervention arm?

See Table 2, row 1 ((the row beginning with "Subjects n"), column 2, N = 318

The control condition was "Placebo".

How many people were in the placebo arm?

See Table 2, row 1 (the row beginning with "Subjects n"), column 3, $N=161$

We will input these numbers in GRADEpro table. You will find all data in Table 4 as that table contains the results on the outcomes.

Here, you have several choices. Read this slowly.

I have already told you that you will need to consider the FINAL data collection point, in case of this article, that FINAL data collection point is Week 52. If you read across the Row Marked Week 52, you will see

44 people remained on "continuous abstinence" at Week 52 (see the column marked Nicotine Mouth Spray).

So, input these numbers in the GRADE table where it says "Summary of findings" in the columns beneath that (Intervention and Control sub-columns, whatever you have named them):

For the intervention, 
total number: 318

number who had the outcome: 44

For the control,

total number: 161

number who had the outcome: 9

Then I have told you in the class several times now that RR stands for Relative Risk or Risk Ratio. From Table 4, we see that

RR: 2.48

95\% Cl: $1.24-4.94$

Plug in these numbers in Gradepro next

AS SOON AS YOU WILL PLUG in these numbers, GRADEPRO will automatically calculate the absolute effect, in this case 83 MORE per 1000 people (this per 1000 people is by default, you can adjust this value, I will leave it to you to work it out how!)

See the screenshot of the summary of findings.

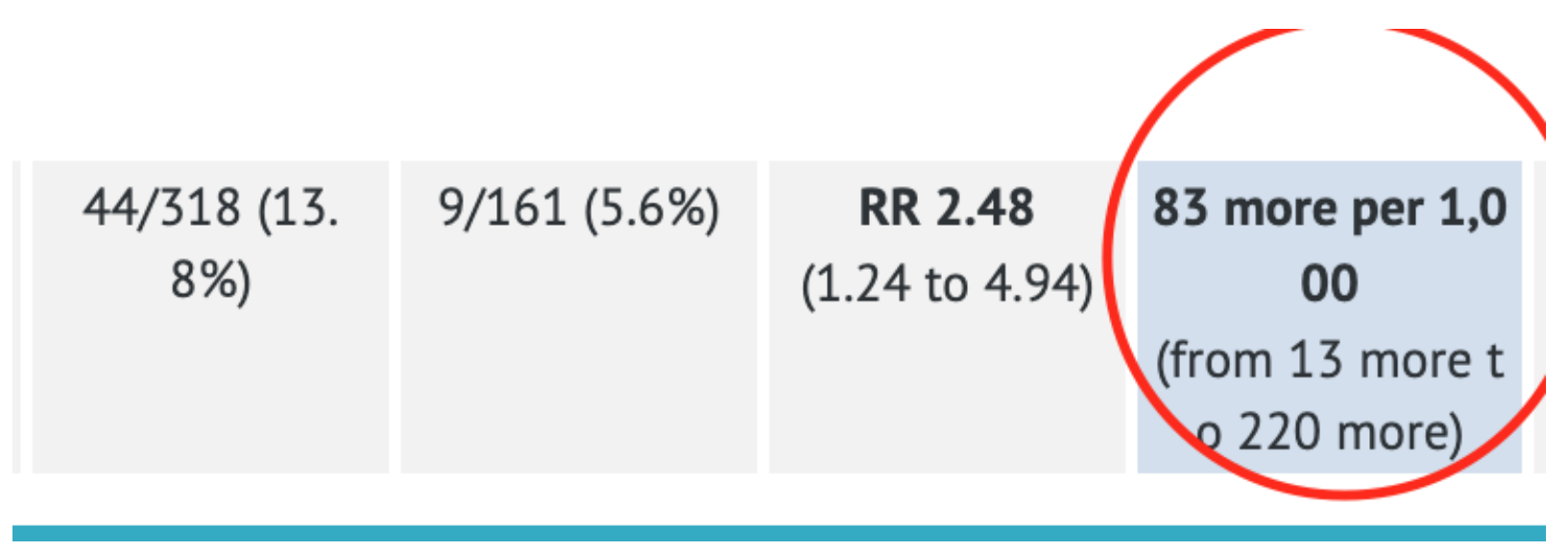

That's all for filling in the Summary of Findings part.

So, basically, to fill in the summary of findings table:

- First learn the concepts of what is RR, what is binary versus continuous variable.

- Next, learn WHAT outcome variable is studied and HOW that outcome is measured. What units, how exactly is it measured.

- In this article, we have discussed how to code it if it's binary and presents data from a randomized controlled trial. In subsequent papers we will expand the scope to what happens when we have other study designs including metaanalyses

- If it's binary, as it was in this case, then pay attention to how many people were in each of treatment and control arm (based on initial enrolment), and how many people experienced the outcome at the LAST or FINAL assessment.

- Identify the appropriate numbers and plug in those numbers to the GRADEpro summary of findings tables.

\section{Conclusion}


In this paper, we presented a way to enter data into GRADEpro software for conducting GRADE based analysis of research data. In subsequent papers, we will discuss how to enter other types of data into GRADE tables to create summary of findings tables.

\section{References}

^David Atkins, The GRADE Working Group, Martin Eccles, Signe Flottorp, Gordon H Guyatt, David Henry. (2004). Systems for grading the quality of evidence and the strength of recommendations I: Critical appraisal of existing approaches The GRADE Working Group. BMC Health Serv Res, vol. 4 (1). doi:10.1186/1472-6963-4-38.

?. ^Philip Tønnesen, Hans Lauri, Roland Perfekt, Karl Mann, Anil Batra. (2012). Efficacy of a nicotine mouth spray in smoking cessation: a randomised, double-blind trial. Eur Respir J, vol. 40 (3), 548-554. doi:10.1183/09031936.00155811. 